
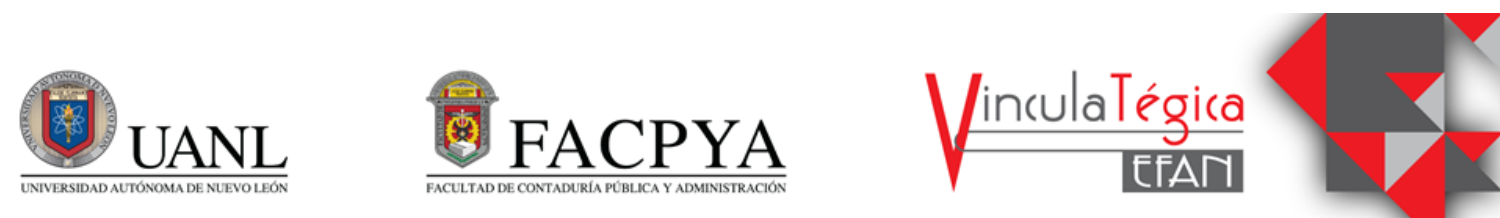

\title{
El efecto del COVID-19 en la percepción del bienestar del individuo y la transición hacia la organización digital
}

\author{
Francisco Javier Álvarez Torres ${ }^{1}$, Miriam Adriana Mendoza Romero² y Davinía Elena Ríos \\ Herrera $^{3}$ \\ 'Universidad de Guanajuato, División de Ciencias Naturales y Exactas, \\ Guanajuato, Guanajuato, México, fjalvarez@ugto.mx, Noria Alta S/N Campus Guanajuato, \\ (+52) 4737320006 \\ ${ }^{2}$ Educación Despierta, adriesmam@gmail.com, La Bufa 7, Guanajuato, Guanajuato, México, \\ (+52) 4731201999 \\ ${ }^{3}$ Universidad de Guanajuato, División de Ciencias Naturales y Exactas, \\ Guanajuato, Guanajuato, México, rh.davinia@gmail.com, Noria Alta S/N Campus Guanajuato, \\ (+52) 4737320006
}

Información del artículo revisado por pares

Fecha de aceptación: junio-2021

Fecha de publicación en línea: diciembre-2021

DOI: https://doi.org/10.29105/vtga7.2-59

\section{Resumen}

La pandemia COVID-19 y el proceso de distanciamiento social que se han vivido en el año 2020 han traído diversos impactos en la percepción del bienestar de las personas y en la adaptación al trabajo remoto. Este fenómeno ha conducido a reflexionar en el diseño de estrategias en el corto y mediano plazo para atender las implicaciones de bienestar, tanto a nivel individual como organizacional, tanto en las personas que adaptaron su trabajo a una modalidad remota, como para aquellas que no pudieron adaptar sus labores y perdieron sus trabajos. En este estudio se encuestaron a 587 personas, quienes describen los cambios de comportamiento, economía familiar, hábitos y dinámicas derivados del proceso de resguardo en casa en los hogares de las familias mexicanas, mayormente del estado de Guanajuato, así como las adecuaciones en la adaptación al trabajo remoto.

\begin{abstract}
The COVID-19 pandemic and the process of social distancing that have been experienced in 2020 have brought various impacts on the perception of people's well-being and on adaptation to remote work. This phenomenon has led to reflection on the design of strategies in the short and medium term to address the implications of well-being, both at the individual and organizational level, both in people who adapted their work to a remote mode, and for those who could not adapt their work and lost their jobs.

In this study, 587 people were surveyed, who describe the changes in behavior, family economy, habits and dynamics derived from the process of safeguarding at home in the homes of Mexican families, mostly in the state of Guanajuato, as well as the adaptations in adaptation to remote work.

The results indicate the emotional, physical and economic affectations for those who
\end{abstract}


Los resultados señalan las afectaciones emocionales, físicas y económicas para aquellos que adaptaron sus condiciones a trabajos remotos, pero también dan la pauta para describir como la percepción de bienestar fue afectada en esta etapa. Para los trabajadores remotos, estos resultados dan pie al diseño de un modelo de transición de un liderazgo exclusivamente pensado en un entorno presencial hacia un liderazgo humano-tecnológico orientado en promover el bienestar del individuo, que de pauta a un mayor autocuidado del bienestar físico y mental por parte del líder en sus colaboradores y que éste sea un eje fundamental en el desarrollo de la productividad y desempeño de sus colaboradores en la organización netamente digital.

Palabras clave: COVID-19, Bienestar, Organización Digital, Liderazgo, Tecnología.

\section{INTRODUCCIÓN}

El bienestar y la salud de las personas es un elemento importante en el desarrollo de las personas, lamentablemente en la mayoría de las ocasiones es olvidado o subvalorado. La enfermedad, los riesgos de complicaciones de enfermedades crónicas, nuevas enfermedades tanto físicas como psicológicas dan forma a nuestras dinámicas sociales, económicas y políticas (Bloom \& Canning, 2003). Entender como el bienestar está vinculado a nuestras capacidades y su efecto directo en el trabajo requiere investigaciones multidisciplinares que permitan abordar estas relaciones desde múltiples puntos de vista entre las que destacan la salud mental y física y su efecto; para nuevos modelos de bienestar.

Hace poco más de un año, como humanidad hemos vivido una nueva dinámica, impensable para los inicios de adapted their conditions to remote jobs, but they also give the guideline to describe how the perception of well-being was affected at this stage. For remote workers, these results give rise to the design of a leadership transition model exclusively thinking of a face-to-face environment towards a humantechnological leadership aimed at promoting the well-being of the individual, which leads to greater self-care of physical well-being and mental leadership on the part of the leader in his collaborators and that this is a fundamental axis in the development of the productivity and performance of his collaborators in the full digital organization.

Keywords: COVID-19, Wellness, digital organization, Leadership, Technology.

JEL: D6.

este nuevo milenio: La pandemia COVID19. Y con ello, hemos puesto en la agenda global el tema de salud (World Economic Forum, 2020), esto ha causado una preocupación global en la prevención de los contagios de este nuevo virus SARS COV 2, es por ello que en la mayoría de los países se pausaron las actividades educativas y comerciales, modificando hábitos y dinámicas de convivencia social, poco a poco entrando en una reclusión voluntaria, que trasgredía a los patrones de vida tradicionales relacionados al bienestar del individuo: el trabajo, la educación, los alimentos, las actividades físicas, la convivencia social. Los costos de esta distancia social han sido muy altos, especialmente para las economías emergentes como la mexicana, pues no todos los trabajos pudieron adaptarse a estas condiciones de trabajo remoto mediado por las Tecnologías de la 
Información y la Comunicación (TIC), las personas que pudieron hacerlo tuvieron que vivir cambios únicos y drásticos en sus estilos de vida y con ello el bienestar percibido.

Este efecto de incertidumbre global se trasladó tanto a las organizaciones, como a los individuos. Diversas dolencias y afectaciones físicas y emocionales como desórdenes del sueño, alimentación y visión fueron reportadas por diversos estudios durante el período por cuarentena por COVID-19 (Dey \& Dey, 2020; Singh et al., 2020).

En ese sentido, el presente trabajo tiene como objetivo principal describir los principales comportamientos relacionados con la percepción del bienestar individual y social en el marco de la crisis mundial sanitaria por el virus COVID-19 que permita dimensionar desde otra perspectiva el trabajo remoto, de quienes pudieron adaptar su labor y una proyección hacia un liderazgo necesario en la futura organización digital.

\section{MARCO TEÓRICO}

\subsection{Percepción del Bienestar y COVID-19.}

La descripción del bienestar en el contexto del COVID-19 es un reto que sin duda nos lleva a reflexionar la relación entre la salud física, mental y la productividad de las personas que han llevado a cabo su labor de manera remota. De acuerdo a las directrices de algunas organizaciones, ante las complicaciones de la pandemia, muchos trabajadores han estado trabajando desde casa, de manera muy frecuente $\sin$ entrenamiento $o$ los instrumentos tecnológicos necesarios. $\mathrm{Y}$ en ese nuevo escenario, una serie de cuestionamientos nacen, por ejemplo: ¿cómo ha impactado esta transición a los hábitos de vida de las personas? ¿ha mejorado o ha empeorado su percepción del bienestar? ¿cómo ha sido su adaptación al trabajo remoto? ¿es necesario un nuevo liderazgo en este contexto remoto-digital? ¿cuáles son los nuevos modelos de bienestar?

Esta serie de preguntas nos llevan a reflexionar acerca del bienestar, su percepción individual y su relación con el trabajo. Y para ello, es necesario definir este concepto. La percepción del bienestar es un constructo complejo y multifactorial, en su tejido histórico es mayormente abordado por la psicología y las ciencias de la conducta; desde estas áreas del conocimiento el bienestar es definido como el balance afectivo, que se obtiene restando la frecuencia de emociones positivas y la frecuencia de emociones negativas y la satisfacción percibida (Diener \& Fujita, 1995). Es decir, la percepción del bienestar es un concepto subjetivo relativo a tres características principales (Wright \& Cropanzano, 2000). La primera de ellas, como un evento fenomenológico, es decir, una persona percibe ese bienestar, cuando ellos piensan estar en una sensación de ese tipo. Diversas investigaciones en este sentido señalan a la percepción del bienestar como la sensación de ser tocado en la íntima existencia (Lundin et al., 2013; Wright \& Cropanzano, 2000). Para Lundin et al; (2013), el fenómeno del bienestar es deducido por tres componentes: 1) $L a$ sensación de la libertad en las elecciones, 2) La sensación de placer y 3) La sensación de cercanía a alguien o a algo. La segunda característica (Wright \& Cropanzano, 2000) involucra condiciones emocionales, es decir, las personas que perciben el bienestar tiene una mayor orientación a las emociones positivas que a las negativas. Tercero, el bienestar se define como una evaluación global no sólo como una parte de la vida, sino como un todo (Diener, 1994).

Si bien, en la discusión teórica revisada el bienestar pareciera un fenómeno individual, algunos autores 
describen como ciertas situaciones sociales y contextuales pueden facilitar o limitar el desarrollo pleno del bienestar y la auto-motivación que lo origina (Lundin et al., 2013; Ryan \& Deci, 2000).

La pandemia por COVID-19 ha sido el cisne negro (Taleb, 2007) del milenio. Ha generado una serie de cambios en las decisiones individuales y colectivas en muy poco tiempo. Si bien motivados por las precauciones en temas de salud, no podemos obviar las implicaciones económicas, de salud mental y educativas que en el marco del período de cuarentena o resguardo en casa se han generado.

Para una parte de la población mundial, el hogar se nos ha convertido en un espacio saturado, en donde la convivencia familiar, salón de escuela y la oficina dialogan en una dinámica 24/7 que ha tenido impactos en el desarrollo emocional de niños, jóvenes (Liu et al., 2020) y para aquellos que adaptaron sus labores a la virtualidad, los trabajadores remotos (Como et al., 2020).

Si bien, la percepción del bienestar ha sido tema analizado en el pasado por parte del mundo del trabajo (Harter et al., 2004; Netz et al., 2005), en el contexto del COVID-19, se convierte en un elemento estratégico y clave para el mantenimiento de tres elementos que dan forma a una comunidad humana: Satisfacción en el trabajo, cuidado de la salud y el desempeño laboral y la crianza de la familia. Elementos que han sido vulnerados de diversas formas en esta crisis sanitaria (Chien-Hung et al., 2017; Möhring et al., 2021; Özmen et al., 2021).

Esta situación de crisis hace palpable la necesidad no solo de replantearnos el tema de bienestar como individuos o sociedades, sino también repensar el liderazgo en las organizaciones este nuevo paradigma (Deloitte, 2020). Un liderazgo digital pareciera ser una opción, si bien un líder que posea amplias habilidades digitales (hard-skills): uso de conectividad, tecnología open-source, dispositivos móviles y plataformas (Damayanti \& Mirfani, 2021) es fundamental en este paradigma, no es lo único. Opciones que equilibren al uso de la tecnología y a la apreciación y significado de ser humano (soft skills) serán en el corto plazo una tendencia en el mundo del trabajo, especialmente en el balance vida-trabajo (Nam, 2014) y en las fronteras de ambos mundos (Currie \& Eveline, 2011) para evitar problemas de salud, emocionales o de intrusión (Adisa et al., 2017).

Un liderazgo humano-tecnológico pareciera una opción que surge, esta propuesta busca equilibrar entre lo humano y lo digital, pero primordialmente es orientado por un profundo y sólido sentido de bienestar y bases éticas en la ejecución de sus acciones y resultados, en la motivación de sus equipos e individuos y en la visión sustentable y digital de las organizaciones del futuro. Esta crisis de la pandemia COVID-19 nos ha enseñado que somos frágiles, a nivel individual y organizacional y en este proceso de aprendizaje acelerado en el que se han vivido adaptaciones extraordinarias de orden tecnológico y de gestión (Bartsch et al., 2020). Es momento de dar una pausa y brindar un tiempo para el análisis y la reflexión para tejer las bases de una transición paulatina hacia un liderazgo humano-tecnológico en el nacimiento de las futuras organizaciones digitales.

\subsection{Bienestar y Trabajo Remoto.}

La relación entre bienestar individual y colectivo dentro de una organización se convierte en un constructo por atender, especialmente para aquellas organizaciones que buscan sobresalir en su industria al desarrollar una ventaja competitiva en su personal (Porter, 2007), 
especialmente en esta época de redefinición del mundo de los negocios (Donthu \& Gustafsson, 2020). Replantear los riesgos laborales que pueden existir desde el trabajo remoto (remote working), los costos médicos de trabajadores saturados, los beneficios del tiempo favorecido en el cuidado físico por parte de los trabajadores y hasta la inversión en infraestructura adecuada para que se cuente con elementos para el autocuidado, se convierte más que nunca en un valor agregado para atraer y mantener talento en una organización.

Esta relación entre trabajo remoto y bienestar deriva en la discusión de dos elementos clave para las organizaciones: 1) la necesidad de estrategias laborales para favorecer el bienestar del personal y 2) una fuerza laboral entrenada con habilidades necesarias para esta economía PosCOVID-19: Adaptabilidad en nuevas habilidades digitales para trabajo remoto, flexiblidad en las cadenas de distribución de productos y servicios y una alta capacidad de resiliencia frente a la incertidumbre. Si estos dos elementos no se encuentran en el mercado laboral de manera natural, fenómenos laborales como el estadounidense pudieran repetirse en otros países (Olmo, 2021).

La adaptabilidad al trabajo remoto ha significado un reto mayúsculo para las organizaciones con estructuras más rígidas, para aquellas donde sus trabajos son mayormente manuales o en donde sus plantillas laborales existen pocos jóvenes, ya que éstos se adaptan más rápido al proceso del trabajo remoto (Brynjolfsson et al., 2020). Para este tipo de organizaciones transitar durante este último año a un esquema remoto ha sido una tarea ardua de inversión en tecnología, fortalecimiento de la capacitación y el rediseño de procesos (Phillips, 2020).
El trabajo remoto ha traído nuevas discusiones al mundo del management hasta su importancia en la transición hacia las ciudades inteligentes (Kylili et al., 2020). Para fines de este trabajo lo hemos dividido en dos vertientes: 1) Sus ventajas / desventajas para el trabajador y 2) La estructura necesaria en la organización en la búsqueda de un balance (vida-trabajo) de quienes se encuentran en esta modalidad. En el primer punto, es posible mencionar la flexibilidad que esta modalidad permite, consecuencias como el ahorro en traslados por parte de trabajadores y hasta la disminución en el impacto ambiental, especialmente en ciudades grandes son algunos de ellos; sin embargo, entre sus desventajas existen impactos negativos en el bienestar físico y emocional de las personas en esta modalidad (Abrams, 2019; Adisa et al., 2017; Mostafa, 2021; Nam, 2014) por ello velar por un equilibrio es vital para la organización. En el segundo punto, las organizaciones se encuentran en un punto de inflexión al interior de sus estructuras. El bienestar de sus trabajadores ha tomado parte en sus agendas estratégicas, especialmente impulsado por las preocupaciones de salud derivadas por la pandemia, por las presiones del trabajo remoto y los costos médicos (Harrison \& Stephens, 2019; Ho, 2017).

Ante esta situación cuestionar la relación entre el trabajo remoto y el bienestar es fundamental para brindar un espacio de balance entre vida-trabajo. Diversas preguntas surgen al respecto en esta postura, especialmente para las organizaciones digitales: ¿la organización deberá contar con una estructura para facilitar la conciliación entre el trabajo remoto con un balance vida-trabajo de sus trabajadores? ¿son los líderes de las organizaciones los actores clave en la atención al bienestar de sus trabajadores? 
¿qué opciones se le deben brindar al trabajador para mejorar su bienestar en el mundo offline? ¿las jornadas laborales de las organizaciones no digitales aplican al mundo del trabajo remoto?

Esta serie de preguntas abren la oportunidad para discutir la relación entre bienestar, trabajo remoto y liderazgos jugarán en el nuevo tablero organizacional en la era PosCovid. Cualquiera que sea esa propuesta consideramos que coincidirán 3 características derivadas de las lecciones derivadas de esta pandemia del COVID19: a) una estructura organizacional horizontal en la toma de decisiones y cooperación (Forman et al., 2020), y b) liderazgo en mandos altos y medios que fomenten el compromiso, la satisfacción laboral y sobre todo orientado a fortalecer el bienestar de los trabajadores remotos (Felstead \& Henseke, 2017) y c) equipos virtuales con un amplio sentido de autonomía e intraemprendimiento en la gestión (Bailey \& Breslin, 2021). Visualizamos un liderazgo, si bien orientado por el uso tecnológico, fundamentado en la relación humana y en la búsqueda y promoción del bienestar en los colaboradores.

\subsection{Bienestar y liderazgo en la transición hacia la organización digital.}

La situación actual ha puesto un enorme reto para los líderes. Lidiar con la incertidumbre y con cuestiones éticas respecto a la salud y la economía han hecho que nos preguntemos: ¿Qué liderazgo nacerá en la época PosCovid? Los liderazgos actuales demandan una serie de habilidades digitales, de gestión, de conocimientos de salud, de comunicación digital y especialmente del termómetro del bienestar en la organización.
Figura 1. Modelo Propuesto acerca del liderazgo humano-tecnológico y su relación con la percepción del bienestar en la organización digital.

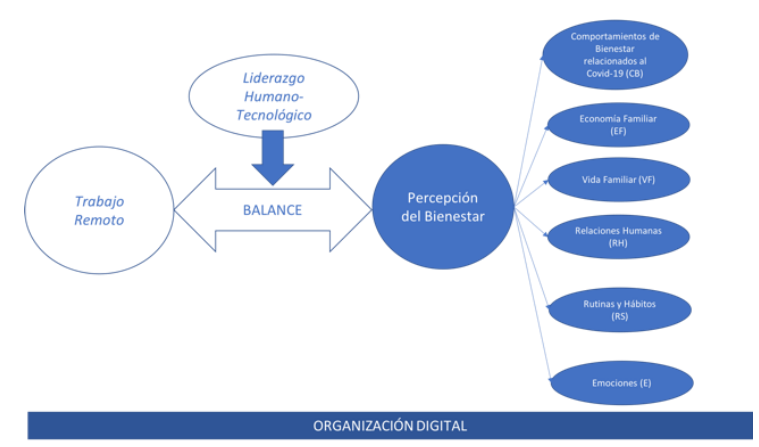

Fuente: Elaboración propia. Para fines de este estudio, se analizan los efectos del COVID-19 en la percepción del bienestar de una muestra.

El modelo que se propone es un liderazgo que transita de un esquema netamente presencial hacia uno en la digitalidad, con un componente orientado a la percepción del bienestar. Lo anterior se deriva de los resultados y la discusión de la investigación en donde la percepción del bienestar ha sido impactada negativamente. Estos hallazgos nos permiten reflexionar que el liderazgo que aparezca después de la recuperación económica, social y de salud requiere amplias habilidades para brindar solidez y estabilidad tanto en aspectos tecnológicos (plataformas, conectividad, hardware, etc) (Kane et al., 2019), pero también en aspectos humanos de sus equipos (balance vida-trabajo, búsqueda espiritual, bienestar físico y mental, etc) (Gallagher, 2019).

Para un liderazgo humano tecnológico dar certidumbre en un entorno altamente volátil requiere de ambientes de cooperación fácil, menor control jerárquico, mayor autonomía de los trabajadores y el diseño de una organización digital que promocione y cuide el bienestar de sus trabajadores 
brindando un ambiente de mayor compromiso (Snow et al., 2017) y del cuidado del balance entre vida y trabajo.

\section{MÉTODO}

Para evaluar los efectos del resguardo en casa derivado de la pandemia en la percepción del bienestar de las personas, las futuras implicaciones en la dinámica trabajo remoto y la posibilidad de un nuevo liderazgo que apareciera en este escenario, se llevó a cabo una encuesta a 587 personas del estado de Guanajuato mayores de edad, que se encontraban aisladas en sus hogares durante el período Agosto-Diciembre 2020. Los ítems fueron adaptados del Proyecto de la Universidad de Texas llamado The Pandemic Project (Pennebaker et al., 2020) y fueron puestos en un formulario para su llenado electrónico. Se compartió por medios digitales a miembros de la sociedad guanajuatense en su mayoría.

Para verificar y validar la información y el modelo para evaluar la percepción del bienestar, se utilizó un sistema de ecuaciones estructurales por mínimos cuadrados parciales (SEM-PLS) (Hair et al., 2010, 2012, 2018; Iacobucci, 2010). El modelo de investigación cuenta con seis dimensiones reflectivas (véase Figura 3) (Becker et al., 2012) y dos variables categóricas referentes al trabajo $y$ al nivel educativo. Estas variables permiten retratar una parte del fenómeno que viven los participantes en sus hogares en este período y discutir los resultados a la luz de las implicaciones futuras en el trabajo remoto y un nuevo liderazgo.

Se atendió que la estructura del instrumento fuera cuidadosa con las sensaciones de los participantes, por ello se incluyó un consentimiento informado del participante, en donde se explican los ítems, alcances y el proceso de respuesta. La intención es que el instrumento fuese un documento informado, breve y conciso para los participantes. Derivado de la revisión teórica (Como et al., 2020; Cotton \& Hart, 2003; Harrison \& Stephens, 2019; Pennebaker et al., 2020) las dimensiones en las cuales se estructuró el instrumento correspondieron a (véase Figura 2):

Figura 2. Modelo Teórico acerca de la Percepción del Bienestar y su relación al adaptación al trabajo remoto en esta pandemia por el COVID-19.

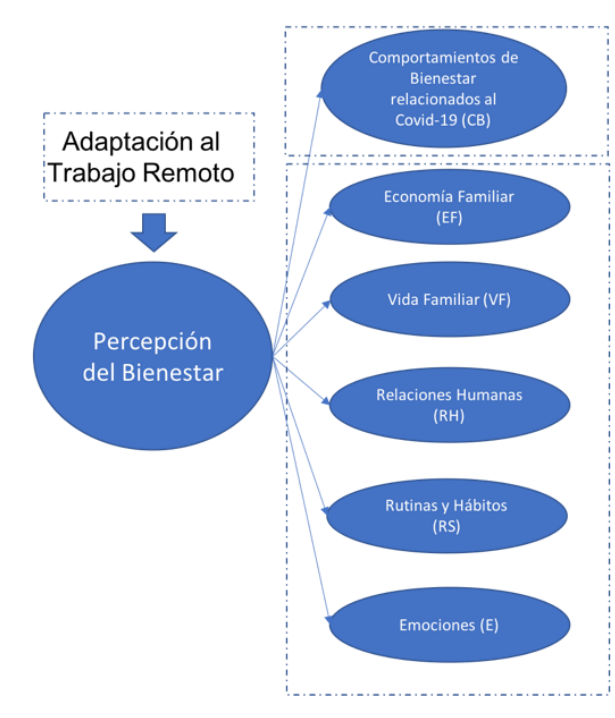

Fuente: Elaboración propia.

Las dimensiones evaluadas se explican a continuación en estas tres categorías:

a) Comportamientos de Bienestar relativos al COVID-19. En esta sección, se llevaron a cabo 11 preguntas acerca de elementos relacionados con el bienestar a partir del escenario del COVID-19, los niveles de preocupación de las personas encuestadas acerca del COVID-19 y el tiempo de permanencia en resguardo. En la primera pregunta, se abre la oportunidad a una respuesta abierta 
en donde las personas expresan: ¿cómo se sienten? En donde se abre la oportunidad de recopilar las historias de las y los participantes.

b) Adaptación al Trabajo Remoto. En esta sección, se llevaron a cabo 10 preguntas orientadoras acerca del tipo de trabajo que tienen, cómo lo han llevado a cabo hasta el momento, si cuentan con opciones adicionales de ingreso y la modalidad en la cual han llevado su trabajo en este tiempo (presencial, mixto o virtual). Esto nos permite conocer el nivel de adecuaciones de los tipos de trabajo que se han hecho por parte de la población encuestada.

c) Hábitos de Bienestar (Economía Familiar, Vida Familiar, Relaciones Humanas, Sociedad, Rutinas y Hábitos y Emociones). En esta sección se llevaron a cabo 43 preguntas orientadoras alrededor de los cambios en comportamiento y hábitos que los participantes han vivido a partir del resguardo en casa en las dimensiones de: Economía Familiar, Vida Familiar, Relaciones Humanas, Sociedad, Rutinas y Hábitos y Emociones. Los resultados de esta sección nos permitieron conocer los cambios en la percepción del bienestar para la población encuestada.

A partir de lo anterior, se derivaron 6 hipótesis para esta investigación que corresponden a describir el constructo de percepción de bienestar y su comportamiento en las dimensiones reflectivas y categóricas (Becker et al., 2012; Simonetto, 2012):
Hipótesis 1. La dimensión de Comportamientos de Bienestar relacionados al COVID-19 se refleja positiva y significativamente en la percepción del bienestar.

Hipótesis 2. La dimensión de Economía Familiar se refleja positiva y significativamente en la percepción del bienestar.

Hipótesis 3. La dimensión de Vida Familiar se refleja positiva $y$ significativamente en la percepción del bienestar.

Hipótesis 4. La dimensión de Relaciones Humanas se refleja positiva y significativamente en la percepción del bienestar.

Hipótesis 5. La dimensión de Rutinas y Hábitos se refleja positiva $y$ significativamente en la percepción del bienestar.

Hipótesis 6. La dimensión de Emociones se refleja positiva $y$ significativamente en la percepción del bienestar.

Figura 3. Modelo Teórico acerca de los efectos del COVID-19 en la Percepción del Bienestar, ítems e hipótesis.

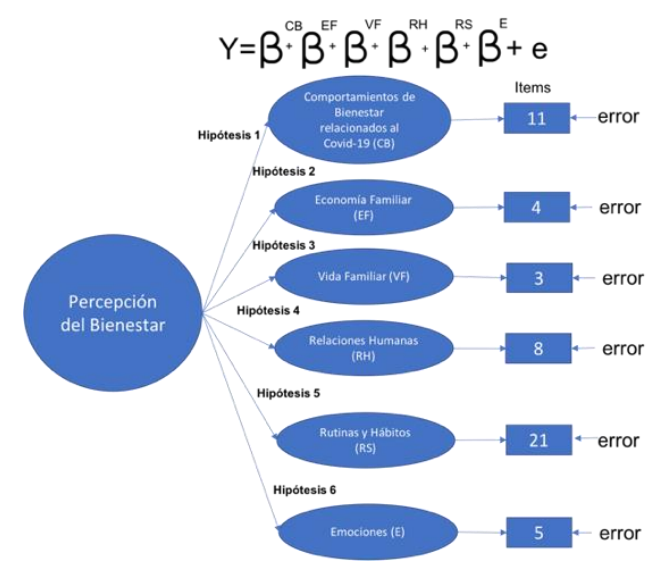


Fuente: Elaboración propia.

La aplicación del algoritmo de PLS-SEM se llevó a cabo en el software SmartPLS ${ }^{\circledR}$ versión 3.3.3 (Ringle et al., 2015), en donde se diseñó el modelo reflectivo tipo I (Becker et al., 2012) para las 587 observaciones.

En el caso de este tipo de modelos, la evaluación inicia con la carga de los indicadores. Las cargas mayores a 0.700 indican que el constructo explica al menos el $50 \%$ de la varianza del indicador, es por ello que se procedió a retirar los indicadores menores a este parámetro.

En la dimensión de comportamientos de bienestar asociados al COVID-19 (CB) fueron retirados los ítems 7A (0.561), 7B (0.499) y 7C (0.556), relativos a medidas de higiene y prevención. En la dimensión de Relaciones Humanas $(\mathrm{RH})$ los ítems que mostraron esta característica fueron $21 \mathrm{~A}(0.569), 21 \mathrm{E}$ (0.615), $21 G$ (0.683). En la dimensión de Rutinas y Hábitos (RS), los ítems retirados correspondieron a $24 \mathrm{~A}$ (0.140), 24B (0.201), 24C (0.509), 24D (0.478), 24E (0.124), 24F (0.347), 25A
(0.269), 25B (0.264), 25C (0.253), 25D (0.312), 25E (0.350), 25F (0.322), 25G (0.294), 26F (0.462), 26G (0.579) y 26H (0.335) relativos a prácticas de manejo del estrés, cambios de percepción de la realidad o cambios en compras y tiempo en línea. En la dimensión de Emociones (E), los ítems 27C (0.584), $27 D \quad(0.369) \quad y \quad 27 \mathrm{E} \quad$ (0.373) correspondientes a la actuación con violencia e irritación fueron retirados por su carga (véase Figura 4).

Figura 4. Modelo Path acerca de la los efectos del COVID-19 en la Percepción del Bienestar.

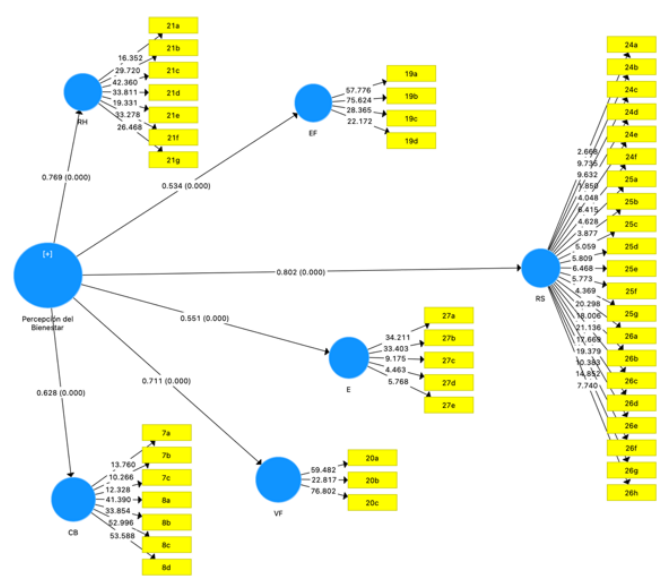

Fuente: Elaboración propia a partir de la información obtenida por SmartPLS®

El próximo paso de la validación del modelo es analizar la confiabilidad de consistencia interna para el uso de SEMPLS se determina a través de la confiabilidad compuesta, la cual determina que para estudios exploratorios los márgenes de 0.600 a 0.700 son 
aceptables, parámetros entre 0.700 y 0.950 son considerados satisfactorios y excelentes y aquellos mayores a 0.950 son problemáticos.

Las dimensiones obtuvieron puntajes mayores a 0.850 y menores a 0.950 (véase tabla 1).

Tabla 1. Ajustes de medida de modelo reflectivo de Percepción del Bienestar.

\begin{tabular}{|c|l|c|c|}
\hline \multicolumn{2}{|c|}{ Constructo / Dimensiones } & $\begin{array}{c}\text { Confiabilidad } \\
\text { compuesta }\end{array}$ & AVE \\
\hline \multirow{5}{*}{} & $\begin{array}{l}\text { Comportamientos } \\
\text { de Bienestar } \\
\text { relacionados al } \\
\text { Covid-19 (CB) }\end{array}$ & 0.925 & 0.755 \\
\cline { 2 - 4 } & Emociones (E) & 0.948 & 0.901 \\
\cline { 2 - 4 } $\begin{array}{c}\text { Percepción } \\
\text { del }\end{array}$ & $\begin{array}{l}\text { Economía Familiar } \\
\text { (EF) }\end{array}$ & 0.873 & 0.633 \\
\cline { 2 - 4 } & $\begin{array}{l}\text { Relaciones } \\
\text { Humanas (RH) }\end{array}$ & 0.852 & 0.591 \\
\cline { 2 - 4 } & $\begin{array}{l}\text { Rutinas y Hábitos } \\
\text { (RS) }\end{array}$ & 0.855 & 0.543 \\
\cline { 2 - 4 } & $\begin{array}{l}\text { Vida Familiar } \\
\text { (VF) }\end{array}$ & 0.858 & 0.670 \\
\hline
\end{tabular}

Fuente: Elaboración propia a partir de la información obtenida por SmartPLS $®$

El siguiente paso de validación corresponde a la validez convergente, la cual esta determinada por la Varianza Promedio Extraída (AVE). Este indicador es calculado por la media del cuadrado de las cargas de todos los indicadores asociados con ese constructo (Sarstedt et al., 2014). Un AVE aceptable corresponde a 0.500 o mayor (véase tabla 1). Posterior de las anteriores pruebas del modelo, es necesario determinar la validez discriminante. Lo anterior determina que tan distinto es un constructo de otros en el modelo. La forma más conservadora de hacerlo es a través del criterio de FornellLarcker (Hair et al., 2014) (véase Tabla 2).

Tabla 2. Criterio de Fornell-Larcker

$$
\begin{array}{llllll}
C B & E & E F & R H & R S & V F
\end{array}
$$

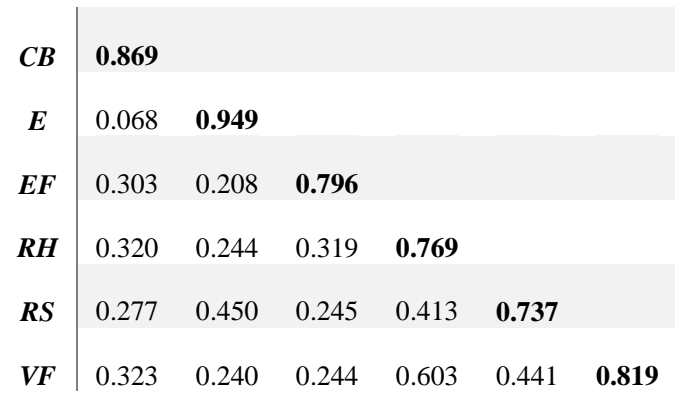

Fuente: Elaboración propia a partir de la información obtenida por SmartPLS ®

\section{La evaluación del modelo} estructural es necesaria. Para ello se evaluan: a) coeficiente de determinación (R2), b) redundancia de validación cruzada (Q2) y c) los coeficientes path (Sarstedt et al., 2014) (véase tabla 3).

Tabla 3. $\mathrm{R}^{2}, \mathrm{R}^{2}$ ajustada $\mathrm{y} \mathrm{Q}^{2}$ del modelo estructural.

\begin{tabular}{r|lll}
\multicolumn{1}{c}{} & \multicolumn{1}{l}{$R^{2}$} & $R^{2}$ & \multicolumn{1}{l}{$Q^{2}$} \\
\hline ajustada & \\
$E$ & 0.342 & 0.340 & 0.249 \\
$E F$ & 0.231 & 0.230 & 0.201 \\
$R H$ & 0.286 & 0.284 & 0.173 \\
$R S$ & 0.582 & 0.581 & 0.335 \\
$V F$ & 0.545 & 0.544 & 0.288 \\
& 0.517 & 0.516 & 0.337
\end{tabular}

Fuente: Elaboración propia a partir de la información obtenida por SmartPLS®

El indicador de $\mathrm{R}^{2}$ es una medida que nos permite predecir los resultados del modelo. Valores entre 0.660 y 0.330 indica un valor explicatorio moderado del modelo. Las dimensiones que poseen esta 
característica corresponde a

Relaciones Humanas (RH), Rútinas y Hábitos (RS) y Vida Familiar (VF). Otra manera de evaluar la relevancia predictiva del modelo como una medida de precisión es $\mathrm{Q}^{2}$, como medida valores mayores a 0 indican un valor predictivo aceptable (Hair et al., 2014; Sarstedt et al., 2014).

Tabla 4. Hipótesis, coeficientes path, valor $\mathrm{t} \mathrm{y}$ significancia del modelo estructural

\begin{tabular}{|c|c|c|c|}
\hline Relaciones e Hipótesis & Coeficiente $P$ & & Valores \\
\hline $\begin{array}{l}\text { Hip. 1. Percepción del } \\
\text { Bienestar -> CB }\end{array}$ & 0.584 & $* * *$ & 15.283 \\
\hline $\begin{array}{l}\text { Hip. 2. Percepción del } \\
\text { Bienestar } \rightarrow \text { E }\end{array}$ & 0.481 & $* * *$ & 10.541 \\
\hline $\begin{array}{l}\text { Hip. 3. Percepción del } \\
\text { Bienestar -> EF }\end{array}$ & 0.534 & $* * *$ & 13.775 \\
\hline $\begin{array}{l}\text { Hip. 4. Percepción del } \\
\text { Bienestar -> RH }\end{array}$ & 0.763 & $* * *$ & 38.164 \\
\hline $\begin{array}{l}\text { Hip. 5. Percepción del } \\
\text { Bienestar -> RS }\end{array}$ & 0.738 & $* * *$ & 32.885 \\
\hline $\begin{array}{l}\text { Hip. 6. Percepción del } \\
\text { Bienestar -> VF }\end{array}$ & 0.719 & $* * *$ & 30.306 \\
\hline
\end{tabular}

Se llevó a cabo el bootstrapping con 5,000 submuestras (Hair et al., 2014) obteniendo seis relaciones entre las variables (véase tabla 4). A través de los resultados puede observarse que la percepción del bienestar es reflejada de manera positiva y significativa con un mayor coeficiente por la dimensiones de Relaciones Humanas (RH) 0.763 , Rutinas y Hábitos (RS) con
0.738 y Vida Familiar (VF) con 0.719. Esto sin duda es un hallazgo en medio de esta pandemia, que tendrá implicaciones en las adecuaciones de bienestar para los trabajadores en el trabajo remoto de las organizaciones netamente digitales.

\section{RESULTADOS}

El modelo y los resultados indica que el proceso de encierro derivado de la cuarentena cambió la percepción del bienestar y las preocupaciones de las personas. De manera descriptiva, el 17\% mencionó sentirse muy preocupado acerca de perder su trabajo y el $40 \%$ mencionó que el encierro derivado de la cuarentena impactó en sus relaciones humanas con su familia, amigos y compañeros de trabajo. De acuerdo a un análisis multi-grupo, las mujeres y personas con un nivel básico de educación reflejaron cambios en la percepción de su bienestar en la dimensión de Economía Familiar (EF).

Los resultados señalan el impacto económico de esta pandemia en las poblaciones con menor nivel educativo y con un sesgo de género, ya que las mujeres participantes en la encuesta fueron las más afectadas en el equilibrio vida-trabajo que los hombres. Esta tendencia corresponde con otros estudios realizados durante la pandemia (Yildirim \& Eslen-Ziya, 2021).

Las dimensiones analizadas tuvieron impactos diferenciados. A manera descriptiva, $19 \%$ de la población encuestada se sintió enojada e irritada y aproximadamente 5\% mencionó haber actuado con violencia o abusar del alcohol o el tabaco. En el análisis de relaciones en 
el modelo se demuestra que los participantes con un mayor nivel educativo reflejan un mayor efecto de esta dimensión en la percepción del bienestar, es decir pudiera inferirse que a mayor nivel educativo los cambios en la dimensión de Emociones poseería una mayor relevancia en la percepción del bienestar del individuo (véase Tabla 5). La regulación emocional ha sido un factor muy importante para la gestión adecuada de trabajo-familia durante esta pandemia (Restubog et al., 2020), especialmente para los trabajadores remotos (Wang et al., 2021).

Este es un hallazgo relevante para la investigación, pudiera inferirse que a mayor educación del trabajador, existe una posibilidad mayor de exposición a labores agotadoras emocionalmente o que requieran capacidades de alta concentración intelectual, por lo que estrategias diferenciadas serían una buena opción de atención. Los trabajadores con mayor nivel educativo desarrollan expectativas en su labor y especialmente en el cuidado que la organización debiera tenerles en su bienestar personal (Zapf, 2002).

Tabla 5. Cargas Factoriales de las dimensiones de Percepción del Bienestar por grupos de nivel educativo.

\begin{tabular}{|c|c|c|}
\hline & ación Básica & $\begin{array}{c}\text { Educación Superior y } \\
\text { Posgrado }\end{array}$ \\
\hline$C B$ & 0.572 & 0.602 \\
\hline$E$ & 0.386 & 0.589 \\
\hline$E F$ & 0.529 & 0.532 \\
\hline$R H$ & 0.744 & 0.788 \\
\hline$R S$ & 0.716 & 0.753 \\
\hline$V F$ & 0.732 & 0.720 \\
\hline
\end{tabular}

Fuente: Elaboración propia a partir de la información obtenida por SmartPLS®
En lo relativo al sentido de comunidad, $35 \%$ de los participantes respondieron que el encierro unirá más a las personas y el $34.75 \%$ mencionó haber ofrecido ayuda a otras personas en este período. Los cambios económicos son la mayor preocupación de los participantes, el $80.6 \%$ mencionó no contar con un ingreso extra durante este período de encierro y el $8.5 \%$ no pudo realizar trabajo remoto de acuerdo a las condiciones de su trabajo y $25 \%$ continuo realizando su trabajo de manera presencial al $100 \%$ (véase Figura 4). Este es un indicador importante del proceso de adaptación de un trabajo netamente presencial a uno remoto.

De acuerdo al análisis descriptivo, el $41.8 \%$ de los participantes mencionaron su capacidad de adaptar su trabajo a la virtualidad, sin embargo el $38.3 \%$ no pudo migrar a un entorno remoto y para el $8.6 \%$ el escenario fue comprometido para su economía, ya que mencionaron haber perdido su trabajo en este período.

Figura 4. Relación de participantes y la modalidad de sus actividades durante el resguardo en casa derivado de la pandemia COVID-19.

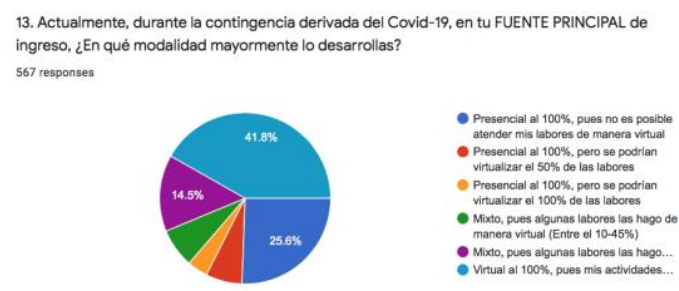

Fuente: Elaboración propia a partir de la información obtenida por SmartPLS $®$

El modelo de relaciones demostró su confiabilidad y validez (véase Tablas 1, 2 y 3) comprobando con ello las hipótesis planteadas a través del modelo 
validado (véase Figura 5). Este es un hallazgo importante, ya que permite un panorama exploratorio de la forma en la que la percepción del bienestar de los individuos fue impactada por el resguardo en casa y la construcción del concepto de lo que es percibido como bienestar en este período.

Si bien la relación entre bienestar y management ha sido abordada $y$ estudiada con anterioridad (PagánCastaño et al., 2020), durante esta pandemia, las medidas de distanciamiento social, las presiones económicas y la tensión emocional que se ha vivido han situado a este concepto con mayor presencia en las agendas de las organizaciones, especialmente para aquellas que buscan transitar a un modelo netamente digital y remoto y que están en búsqueda de la formación y atracción de líderes que entiendan este nuevo paradigma.

Figura 5. Modelo Path validado acerca de la los efectos del COVID-19 en la Percepción del

Bienestar.

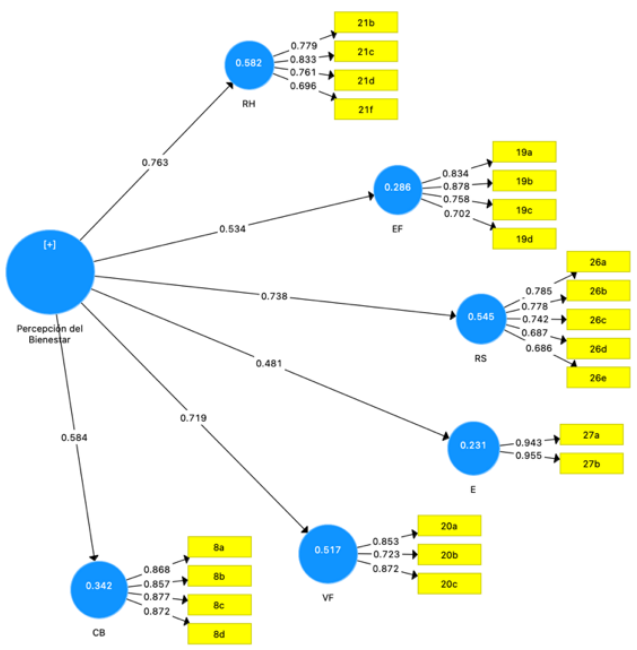

Fuente: Elaboración propia a partir de la información obtenida por SmartPLS $®$

El modelo validado en este estudio describe a la percepción del bienestar como una variable que puede ser medida e interpretada por este concepto con mayor presencia en las agendas de las organizaciones, especialmente para aquellas que buscan transitar a un modelo netamente digital y remoto.

Para la población encuestada los elementos que les permiten contar con una percepción de su bienestar individual se reflejan mayormente en las dimensiones de: Rutina y Hábitos (RS), la Vida Familiar (VF) y las Relaciones Humanas (RH) (Véase Tabla 4). Esto es un hallazgo que tendrá implicaciones en la recuperación económica y organizacional en la era pos-pandemia. Estos resultados proveerán de información para el diseño de presentes y futuras estrategias para aminorar los impactos en estas dimensiones.

Tabla 6. Comprobación de Hipótesis.

\begin{tabular}{|c|c|c|c|c|}
\hline \multicolumn{2}{|c|}{ Hipótesis } & \multicolumn{2}{|c|}{$\begin{array}{c}\text { Coeficiente } \\
\text { Path }\end{array}$} & \multirow{2}{*}{$\begin{array}{l}\text { Valor } t \\
15.283\end{array}$} \\
\hline $\begin{array}{r}\text { Hipótesis } 1 . \\
->\mathrm{CB}\end{array}$ & $\begin{array}{l}\text { Comprobada la } \\
\text { manifestación } \\
\text { positiva y } \\
\text { significativa }\end{array}$ & 0.584 & $* * *$ & \\
\hline $\begin{array}{r}\text { Hipótesis } 2 . \\
->\mathrm{E}\end{array}$ & $\begin{array}{l}\text { Comprobada la } \\
\text { manifestación } \\
\text { positiva y } \\
\text { significativa }\end{array}$ & 0.481 & $* * *$ & 10.541 \\
\hline $\begin{array}{r}\text { Hipótesis } 3 . \\
->E F\end{array}$ & $\begin{array}{l}\text { Comprobada la } \\
\text { manifestación } \\
\text { positiva y } \\
\text { significativa }\end{array}$ & 0.534 & $* * *$ & 13.775 \\
\hline $\begin{array}{r}\text { Hipótesis } 4 . \\
->\mathrm{RH}\end{array}$ & $\begin{array}{l}\text { Comprobada la } \\
\text { manifestación } \\
\text { positiva y } \\
\text { significativa }\end{array}$ & 0.763 & $* * *$ & 38.164 \\
\hline $\begin{array}{r}\text { Hipótesis } 5 . \\
->\text { RS }\end{array}$ & $\begin{array}{l}\text { Comprobada la } \\
\text { manifestación } \\
\text { positiva y } \\
\text { significativa }\end{array}$ & 0.738 & $* * *$ & 32.885 \\
\hline $\begin{array}{r}\text { Hipótesis } 6 . \\
->V F\end{array}$ & $\begin{array}{l}\text { Comprobada la } \\
\text { manifestación } \\
\text { positiva y } \\
\text { significativa }\end{array}$ & 0.719 & $* * *$ & 30.306 \\
\hline
\end{tabular}




\section{CONCLUSIONES}

En resumen, el encierro ha generado diversas condiciones emocionales entre las personas, especialmente en la percepción del bienestar. Cambios en las dinámicas de trabajo y en la adaptación al trabajo remoto mediado por la digitalidad han sido los retos que se han tenido que enfrentar las organizaciones en la inmediatez. Sin embargo, las implicaciones que se derivan de tendrán impactos a largo plazo.

Las conclusiones de este trabajo de investigación girán en torno a tres puntos principales, el primero de ellos:

1) El bienestar: un punto clave en el trabajo remoto. La promoción de hábitos del bienestar físico y mental son fundamental en las organizaciones del futuro, especialmente para los trabajadores remotos, la adaptación hacia la digitalidad implica resiliencia (Hite \& McDonald, 2020), espacio para el cuidado de las emociones y para el mantenimiento de las relaciones humanas. En este nuevo paradigma el desarrollo de un liderazgo con un enfoque orientado a cuidar el bienestar y minimizar la atención tecnológica será un elemento latente en la formación de los nuevos ejecutivos. Las organizaciones que logren este proceso de integración en su misión, visión y valores desarrollarán una ventaja competitiva entre sus competidores. Retomando un valor a la relación humana desde la dignidad en el trabajo y no simplemente por el valor de mercado, uno de los dogmas del hipercapitalismo destructivo que nos enfrentamos en esta era (ByungChul, 2017).

2) El liderazgo humanotecnológico: Dignidad y Balance. Los retos implican nuevas habilidades de liderazgo en entornos digitales, la inclusión educativa en el mundo digital, especialmente para las economías emergentes y el equilibrio vida-trabajobienestar en entornos netamente digitales (Felstead \& Henseke, 2017). Este equilibrio manifiesta la imperiosa necesidad sobre un nuevo liderazgo que emerja en este paradigma. La propuesta denominativa de liderazgo humanotecnológico busca ser un espacio de unión de habilidades que permita una transición de organizaciones netamente digitales con un alto sentido de la dignidad humana de sus colaboradores, líderes y clientes. Un ecosistema primeramente humano, antes que tecnológico; una oportunidad para recuperar un uso distinto de la vida, del trabajo que permite un uso lúdico y contemplativo que no se ahogue simplemente en el hacer (Byung-Chul, 2020), sino en el fomento del ser. Imaginar un líder que promueva una relación humana-tecnológica balanceada entre la relación de trabajo y el autocuidado de los trabajadores con un alto sentido de dignidad en la organización digital pareciera utópico, sin embargo, es un modelo al que paulatinamente debiéramos orientarnos.

3) Organización Digital: Trabajo Remoto y Liderazgo. Las organizaciones digitales se desarrollan en cuanto a complejidad y a cantidad en los últimos años, sin duda la pandemia permitió impulsar a más servicios digitales en la vida diaria. Integrar en estas organizaciones la parte tecnológica en balance con un liderazgo con un alto sentido de balance vida-trabajo podrá orientar mejores resultados en el mediano plazo.

La transición hacia las organizaciones digitales necesita líderes que se adapten a estos nuevos contextos y respondan con la capacidad de armonizar 
entre tecnología y la transición hacia una organización más humana, consciente de la dignidad y socialmente interconectada con otras organizaciones de forma local y global. 


\section{REFERENCIAS}

Abrams, J. (2019). How remote working can increase stress and reduce well-being. The Conversation.

Adisa, T. A., Gbadamosi, G., \& Osabutey, E. L. C. (2017). What happened to the border? The role of mobile information technology devices on employees' work-life balance. Personnel Review, 46(8), 1651-1671. https://doi.org/10.1108/PR-08-2016-0222

Bailey, K., \& Breslin, D. (2021). The COVID-19 Pandemic: What can we learn from past research in organizations and management? International Journal of Management Reviews, 23(1), 3-6. https://doi.org/10.1111/ijmr.12237

Bartsch, S., Weber, E., Büttgen, M., \& Huber, A. (2020). Leadership matters in crisis-induced digital transformation: how to lead service employees effectively during the COVID-19 pandemic. Journal of Service Management, 32(1), 71-85. https://doi.org/10.1108/JOSM-05-2020-0160

Becker, J. M., Klein, K., \& Wetzels, M. (2012). Hierarchical Latent Variable Models in PLS-SEM: Guidelines for Using Reflective-Formative Type Models. Long Range Planning, 45(5-6), 359394. https://doi.org/10.1016/j.lrp.2012.10.001

Bloom, D., \& Canning, D. (2003). Health as Human Capital and its Impact on Economic Performance. Geneva Papers on Risk and Insurance: Issues and Practice, 28(2), 304-315. https://doi.org/10.1111/1468-0440.00225

Brynjolfsson, E., Horton, J. J., Ozimek, A., Rock, D., Sharma, G., \& TuYe, H. (2020). Covid-19 and Remote Work: an Early Look At Us Data. Climate Change 2013 - The Physical Science Basis, June 220, 1-30. https://github.com/johnjosephhorton/remote work/.\%0Ahttp://www.nber.org/papers/w27344\%0ANATIONAL

Byung-Chul, H. (2017). La sociedad del cansancio (Herder (ed.); 1a ed.).

Byung-Chul, H. (2020). La desaparición de los rituales (1a ed.). Herder.

Chien-Hung, Chen, I.-S., \& Chen, J.-C. (2017). A Study Into the Impact of Employee Wellness and Job Satisfaction on Job Performance. The International Journal of Organizational Innovation, 10(October 2017), 252-270.

Como, R., Hambley, L., \& Domene, J. (2020). An Exploration of Work-Life Wellness and Remote Work During and Beyond COVID-19. Canadian Journal of Career Development, 20(1).

Cotton, P., \& Hart, P. M. (2003). Occupational wellbeing and performance: A review of organisational health research. Australian Psychologist, 38(2), 118-127. https://doi.org/10.1080/00050060310001707117

Currie, J., \& Eveline, J. (2011). E-technology and work/life balance for academics with young children. Higher Education, 62(4), 533-550. https://doi.org/10.1007/s10734-010-9404-9

Damayanti, F. P., \& Mirfani, A. M. (2021). An Analysis of Digital Leadership in the Pandemic Covid19 Era. Proceedings of the 4th International Conference on Research of Educational Administration and Management (ICREAM 2020), 526(Icream 2020), 156-159. https://doi.org/10.2991/assehr.k.210212.033

Deloitte. (2020). Leadership in the Time of COVID-19.

Dey, S., \& Dey, I. (2020). Health concerns during lockdown: an observational study among adults of West Bengal. International Journal Of Community Medicine And Public Health, 7(9), 3674. https://doi.org/10.18203/2394-6040.ijcmph20203942

Diener, E. (1994). Assessing subjective well-being: Progress and opportunities. Social Indicators Research, 31(2), 103-157. https://doi.org/10.1007/BF01207052

Diener, E., \& Fujita, F. (1995). Resources, Personal Strivings, and Subjective Well-Being: A Nomothetic and Idiographic Approach. Journal of Personality and Social Psychology, 68(5), 926-935. https://doi.org/10.1037/0022-3514.68.5.926

Donthu, N., \& Gustafsson, A. (2020). Effects of COVID-19 on business and research. Journal of Business Research, 117(June), 284-289. https://doi.org/10.1016/j.jbusres.2020.06.008

Felstead, A., \& Henseke, G. (2017). Assessing the growth of remote working and its consequences for effort, well-being and work-life balance. New Technology, Work and Employment, 32(3), 
195-212. https://doi.org/10.1111/ntwe.12097

Forman, R., Atun, R., McKee, M., \& Mossialos, E. (2020). 12 Lessons learned from the management of the coronavirus pandemic. Health Policy, 124(6), 577-580. https://doi.org/10.1016/j.healthpol.2020.05.008

Gallagher, J. (2019). Indigenous approaches to health and wellness leadership: A BC First Nations perspective. Healthcare Management Forum, 32(1), 5-10. https://doi.org/10.1177/0840470418788090

Hair, J. F., Sarstedt, M., Hopkins, L., G. Kuppelwieser, V., Kuppelwieser, V. G., \& G. Kuppelwieser, V. (2014). Partial least squares structural equation modeling (PLS-SEM). European Business Review, 26(2), 106-121. https://doi.org/10.1108/EBR-10-2013-0128

Hair, J. F., Sarstedt, M., Ringle, C. M., \& Gudergan, S. P. (2018). Advanced Issues in Partial Least Squares Structural Equation Modeling (S. Publications (ed.); 1ra ed.). SAGE Publications. https://doi.org/1483377393

Hair, J. F., Sarstedt, M., Ringle, C. M., \& Mena, J. A. (2012). An assessment of the use of partial least squares structural equation modeling in marketing research. Journal of the Academy of Marketing Science, 40(3), 414-433. https://doi.org/10.1007/s11747-011-0261-6

Hair, J. F., Tatham, R. L., Anderson, R. E., \& Black, W. (2010). Multivariate Data Analysis (Pearson (ed.); 7th ed.).

Harrison, M. A., \& Stephens, K. K. (2019). Shifting From Wellness at Work to Wellness in Work: Interrogating the Link Between Stress and Organization While Theorizing a Move Toward Wellness-in-Practice. Management Communication Quarterly, 33(4), 616-649. https://doi.org/10.1177/0893318919862490

Harter, J. K., Schmidt, F. L., \& Keyes, C. L. M. (2004). Well-being in the workplace and its relationship to business outcomes: A review of the Gallup studies. Flourishing: Positive Psychology and the Life Well-Lived., 205-224. https://doi.org/10.1037/10594-009

Hite, L. M., \& McDonald, K. S. (2020). Careers after COVID-19: challenges and changes. Human Resource Development International, 23(4), 1-11. https://doi.org/10.1080/13678868.2020.1779576

Ho, S. (2017). The future of workplace wellness programs. Strategic HR Review, 16(1), 2-6. https://doi.org/10.1108/shr-11-2016-0101

Iacobucci, D. (2010). Structural equations modeling: Fit Indices, sample size, and advanced topics. Journal of Consumer Psychology, 20(1), 90-98. https://doi.org/10.1016/j.jcps.2009.09.003

Kane, G. C., Phillips, A. N., Copulsky, J., \& Andrus, G. (2019). How digital leadership is(n’t) different. MIT Sloan Management Review, 60(3), 34-39.

Kylili, A., Afxentiou, N., Georgiou, L., Panteli, C., Morsink-Georgalli, P. Z., Panayidou, A., Papouis, C., \& Fokaides, P. A. (2020). The role of Remote Working in smart cities: lessons learnt from COVID-19 pandemic. Energy Sources, Part A: Recovery, Utilization and Environmental Effects, OO(00), 1-16. https://doi.org/10.1080/15567036.2020.1831108

Liu, J. J., Bao, Y., Huang, X., Shi, J., \& Lu, L. (2020). Mental health considerations for children quarantined because of COVID-19. The Lancet Child and Adolescent Health, 4(5), 347-349. https://doi.org/10.1016/S2352-4642(20)30096-1

Lundin, A., Berg, L. E., \& Muhli, U. H. (2013). Feeling existentially touched-A phenomenological notion of the well-being of elderly living in special housing accommodation from the perspective of care professionals. International Journal of Qualitative Studies on Health and Well-Being, 8(1), 1-8. https://doi.org/10.3402/qhw.v8i0.20587

Möhring, K., Naumann, E., Reifenscheid, M., Wenz, A., Rettig, T., Krieger, U., Friedel, S., Finkel, M., Cornesse, C., \& Blom, A. G. (2021). The COVID-19 pandemic and subjective well-being: longitudinal evidence on satisfaction with work and family. European Societies, 23(S1), S601S617. https://doi.org/10.1080/14616696.2020.1833066

Mostafa, B. A. (2021). The Effect of Remote Working on Employees Wellbeing and Work-Life Integration during Pandemic in Egypt. International Business Research, 14(3), 41. 
https://doi.org/10.5539/ibr.v14n3p41

Nam, T. (2014). Technology Use and Work-Life Balance. Applied Research in Quality of Life, 9(4), 1017-1040. https://doi.org/10.1007/s11482-013-9283-1

Netz, Y., Wu, M. J., Becker, B. J., \& Tenenbaum, G. (2005). Physical activity and psychological well-being in advanced age: A meta-analysis of intervention studies. Psychology and Aging, 20(2), 272-284. https://doi.org/10.1037/0882-7974.20.2.272

Olmo, G. D. (2021). Por qué muchos negocios en Estados Unidos no encuentran empleados para cubrir sus vacantes. BBC News. https://www.bbc.com/mundo/noticias-internacional-56780592

Özmen, S., Özkan, O., Özer, Ö., \& Yanardağ, M. Z. (2021). Investigation of COVID-19 Fear, WellBeing and Life Satisfaction in Turkish Society. Social Work in Public Health, 36(2), 164-177. https://doi.org/10.1080/19371918.2021.1877589

Pagán-Castaño, E., Maseda-Moreno, A., \& Santos-Rojo, C. (2020). Wellbeing in work environments. Journal of Business Research, 115(December), 469-474. https://doi.org/10.1016/j.jbusres.2019.12.007

Pennebaker, J., Ashokummar, A., \& Vergani, L. (2020). The Pandemic Project. https://utpsyc.org/covid19/index.html

Phillips, S. (2020). Working through the pandemic: Accelerating the transition to remote working. Business Information Review, 37(3), 129-134. https://doi.org/10.1177/0266382120953087

Porter, M. . (2007). Ventaja Competitiva: Creación y sostenimiento de un desempeño superior (6ta ed.). Grupo Editorial Patria.

Restubog, S. L. D., Ocampo, A. C. G., \& Wang, L. (2020). Taking control amidst the chaos: Emotion regulation during the COVID-19 pandemic. Journal of Vocational Behavior, 119(May), 1-6. https://doi.org/10.1016/j.jvb.2020.103440

Ringle, C. M., Wende, S., \& Becker, J. M. (2015). Smart PLS 3 (3.2.7). http://www.smartpls.com.

Ryan, R. M., \& Deci, E. L. (2000). Self-determination theory and the facilitation of intrinsic motivation, social development, and well-being. American Psychologist, 55(1), 68-78.

Sarstedt, M., Ringle, C. M., Smith, D., Reams, R., \& Hair, J. F. (2014). Partial least squares structural equation modeling (PLS-SEM): A useful tool for family business researchers. Journal of Family Business Strategy, 5(1), 105-115. https://doi.org/10.1016/j.jfbs.2014.01.002

Simonetto, A. (2012). Formative and reflective models: State of the art. Electronic Journal of Applied Statistical Analysis, 5(3), 452-457. https://doi.org/10.1285/i20705948v5n3p452

Singh, S., Roy, D., Sinha, K., Parveen, S., Sharma, G., \& Joshi, G. (2020). Impact of COVID-19 and lockdown on mental health of children and adolescents: A narrative review with recommendations. Psychiatry Research, 293(January).

Snow, C. C., Fjeldstad, Ø. D., \& Langer, A. M. (2017). Designing the digital organization. Journal of Organization Design, 6(1), 7-8. https://doi.org/10.1186/s41469-017-0017-y

Taleb, N. N. (2007). The black swan: the impact of the highly improbable. Random House.

Wang, B., Liu, Y., Qian, J., \& Parker, S. K. (2021). Achieving Effective Remote Working During the COVID-19 Pandemic: A Work Design Perspective. Applied Psychology, 70(1), 16-59. https://doi.org/10.1111/apps.12290

World Economic Forum. (2020). These are the 10 biggest global health threats of the decade. https://www.weforum.org/agenda/2020/02/who-healthcare-challenges-2020s-climate-conflictepidemics/

Wright, T. A., \& Cropanzano, R. (2000). Psychological well-being and job satisfaction as predictors of job performance. Journal of Occupational Health Psychology, 5(1), 84-94. https://doi.org/10.1037/1076-8998.5.1.84

Yildirim, T. M., \& Eslen-Ziya, H. (2021). The differential impact of COVID-19 on the work conditions of women and men academics during the lockdown. Gender, Work and Organization, 28(S1), 243-249. https://doi.org/10.1111/gwao.12529

Zapf, D. (2002). Emotion work and psychological well-being. A review of the literature and some conceptual considerations. Human Resource Management Review, 12(2), 237-268. 
https://doi.org/10.1016/S1053-4822(02)00048-7 